\title{
$T$-matrix method and its applications to electromagnetic scattering by particles: A current perspective
}

\author{
Michael I. Mishchenko ${ }^{\mathrm{a}, *}$, Larry D. Travis ${ }^{\mathrm{a}}$, Daniel W. Mackowski ${ }^{\mathrm{b}}$ \\ a NASA Goddard Institute for Space Studies, 2880 Broadway, New York, NY 10025, USA \\ ${ }^{\mathrm{b}}$ Department of Mechanical Engineering, 201 Ross Hall, Auburn University, AL 36849-5341, USA
}

\section{A R T I C L E I N F O}

\section{Article history:}

Received 25 January 2010

Accepted 26 January 2010

Keywords:

Electromagnetic scattering

$T$-matrix method

Nonspherical particles

Radiative transfer

Coherent backscattering

Optical particle characterization

Remote sensing

\begin{abstract}
A B S T R A C T
This note serves as a short introduction to the reprint of our article " $T$-matrix computations of light scattering by nonspherical particles: a review" (JQSRT 1996; 55:535-75). We first discuss the motivation for writing that article and explain its historical context. This is followed by a short overview of more recent developments.
\end{abstract} Published by Elsevier Ltd.
Although the T-matrix method was formally introduced by P.C. Waterman in 1965 [1], its widespread use and popularity can be attributed equally to his seminal 1971 article [2] published in Physical Review D (Fig. 1). These papers have been cited 350 and 520 times, respectively, in publication outlets covered by the ISI Web of Knowledge ${ }^{\mathrm{SM}}$ and can both be credited with the subsequent use of the $T$-matrix approach in more than 1450 peer-reviewed publications on electromagnetic scattering by finite particles alone [3-6] (Fig. 2). An important role in the popularization of the T-matrix method during the 1970s and 1980s belonged to the 1975 paper by Barber and Yeh [7] published in a more traditional optical journal and cited 340 times.

The conceptual scope of a $T$-matrix has expanded quite dramatically since it was first introduced in Refs. [1,2]. From being a mere bi-product of the extended boundary condition method (otherwise known as the null-field method), the $T$-matrix has become the centerpiece of a

\footnotetext{
* Corresponding author. Tel.: +1 212678 5590; fax: +1 2126785622.

E-mail address: mmishchenko@giss.nasa.gov (M.I. Mishchenko).
}

vast domain of science dealing with electromagnetic, acoustic, and elastic wave scattering [8]. In particular, the advent of the superposition $T$-matrix concept in Refs. [9-11] allows one to give the following general definition.

In the $T$-matrix method, the incident and scattered electric fields are expanded in series of suitable (vector) spherical wave functions, and the relation between the columns of the respective expansion coefficients is established by means of a transition matrix (or $T$-matrix). This concept can be applied to the entire scatterer as well as to separate parts of a composite scatterer.

By 1995, the $T$-matrix method had become the subject of several specialized monographs [12-17] and a sizable body of peer-reviewed journal publications on electromagnetic scattering (Fig. 2). Hence there was a perception that this field was ripe for a review paper, which was, in fact, solicited by the organizer of the first conference on "Light Scattering by Non-Spherical Particles", Joop Hovenier, for his special issue of JQSRT [18]. Our review [19] was published in 1996 and turned out to be timely indeed. It has been cited more than 300 times and thereby has contributed to the subsequent rapid growth of popularity 


\section{Symmetry, Unitarity, and Geometry in Electromagnetic Scattering*}

P. C. WATERMAN

The MITRE Corporation, Bedford, Massachusetts 01730

(Received 11 August 1970)

Upon defining vector spherical partial waves $\left\{\boldsymbol{\psi}_{n}\right\}$ as a basis, a matrix equation is derived describing scattering for general incidence on objects of arbitrary shape. With no losses present, the scattering matrix is then obtained in the symmetric, unitary form $S=-\hat{Q}^{\prime *} \hat{Q}^{*}$, where (perfect conductor) $\hat{Q}$ is the Schmidt orthogonalization of $Q_{n n^{\prime}}=(k / \pi) \int d \sigma \cdot\left[\left(\nabla \times \operatorname{Re} \psi_{n}\right) \times \psi_{n^{\prime}}\right]$, integration extending over the object surface. For quadric (separable) surfaces, $Q$ itself becomes symmetric, effecting considerable simplification. A secular equation is given for constructing eigenfunctions of general objects. Finally, numerical results are presented and compared with experimental measurements.

Fig. 1. Abstract of Ref. [2].

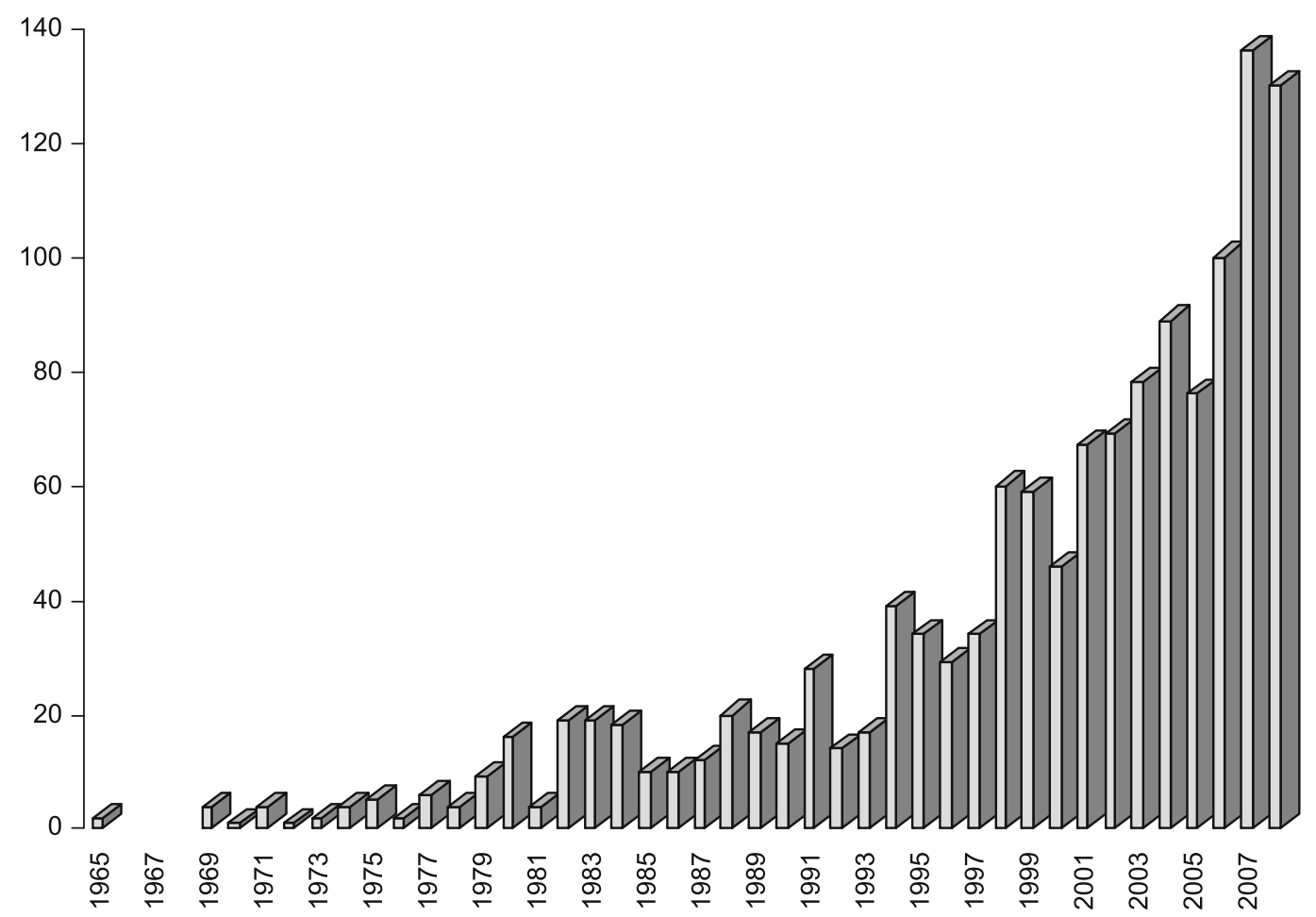

Fig. 2. Annual frequency distribution of peer-reviewed $T$-matrix publications dealing with electromagnetic scattering by finite particles.

of the T-matrix method (Fig. 2). Quite remarkably, two more papers presented at that 1995 conference in Amsterdam and published in Ref. [18] are also among the 17 milestone JQSRT papers featured in this anniversary issue [20,21].

Among the many advances since 1996, perhaps the most significant has been the extension of the analytical orientation-averaging procedure developed in Ref. [22] to the case of an arbitrary multi-sphere cluster in the framework of the superposition $T$-matrix method. Indeed, the resulting paper [23] has been cited 190 times, and its citation rate has been increasing rather than decreasing over time. Another fundamental advance has been the development of the null-field method with discrete sources [24]. From the standpoint of a practical user of the $T$-matrix method, an important tool has been the public-domain package of FORTRAN computer programs available at http://www.giss.nasa.gov/staff/mmishchenko/t_matrix.html. This package is based on developments summarized in Refs. $[22,23,25]$ and has been used in more than 600 peer-reviewed publications. A host of other $T$-matrix programs are available at the web site http://www.scattport.org maintained by Thomas Wriedt.

The range of research areas dealing with the $T$-matrix method and its applications has been quite broad and has included the following:

- Modifications and generalizations of the extended boundary condition method.

- Electromagnetic scattering by anisotropic, bi-isotropic, and chiral objects. 
- Modifications and generalizations of the superposition T-matrix method.

- Electromagnetic scattering by periodic arrays of particles and photonic crystals.

- Electromagnetic scattering by discrete random media.

- Symmetry properties of the $T$-matrix and analytical ensemble-averaging approaches.

- Convergence and efficiency of various implementations of the $T$-matrix method.

- Calculations for various rotationally symmetric particles.

- Calculations for ellipsoids, polyhedral scatterers, and other particles lacking axial symmetry.

- Calculations for layered and composite particles.

- Calculations for clusters of homogeneous and layered spheres.

- Calculations for clusters of nonspherical monomers.

- Calculations for particles with one or several inclusions.

- Theory and calculations of optical resonances in nonspherical particles and particle clusters.

- Theory and calculations of optical forces and torques on small particles.

- Theory and calculations of internal, surface, and local fields.

- Illumination by focused beams and non-plane waves.

- Theory and calculations of electromagnetic scattering by particles near infinite interfaces.

- Computations of anisotropic and aggregation properties of colloids and other disperse media.

- Use of $T$-matrix calculations for testing other theoretical techniques, including effective medium approximations.

- Comparisons of $T$-matrix and controlled laboratory results; analyses of laboratory data.

- Modeling of scattering properties of mineral aerosols and soil particles.

- Modeling of scattering properties of carbonaceous and soot aerosols and soot-containing aerosol and cloud particles.

- Modeling of scattering properties of cirrus cloud particles and hydrometeors.

- Modeling of scattering properties of terrestrial stratospheric and mesospheric aerosol and cloud particles.

- Modeling of scattering properties of aerosol and cloud particles in planetary atmospheres.

- Modeling of scattering properties of interstellar, interplanetary, and cometary dust, planetary regoliths, and planetary ring particles.

- Modeling of absorption and scattering by particles in combustion systems.

- Modeling of scattering and absorption properties of microorganisms, cells, and tissues.

- Various industrial and military applications.

There are, of course, unsolved problems and challenges in the $T$-matrix approach. Two obvious new directions that one can think of would be:

- scattering of pulses, e.g., femtosecond laser scattering; and
- inverse problems: given a $T$-matrix, what information can be gathered regarding the properties of the scatterer?

The volume and extent of new research results are such that now it would be extremely difficult and perhaps impracticable to summarize them in one comprehensive review article or even in a monograph. The specialized monographs [24-30] and topical reviews [31-40] should be a good starting point for a newcomer interested in the theory of the $T$-matrix method and/or its applications. The multi-part reference database [3-6] should also be helpful by providing a classification of all $T$-matrix publications on electromagnetic scattering into narrower subject categories similar to those listed above.

\section{Acknowledgments}

We thank Joop Hovenier, Michael Kahnert, Nikolai Khlebtsov, M. Pinar Mengüç, Victor Tishkovets, and Gorden Videen for numerous useful discussions. This project was sponsored by the NASA Radiation Sciences Program managed by Hal Maring.

\section{References}

[1] Waterman PC. Matrix formulation of electromagnetic scattering Proc IEEE 1965;53:805-12.

[2] Waterman PC. Symmetry, unitarity, and geometry in electromagnetic scattering. Phys Rev D 1971;3:825-39.

[3] Mishchenko MI, Videen G, Babenko VA, Khlebtsov NG, Wriedt T. $T$-matrix theory of electromagnetic scattering by particles and its applications: a comprehensive reference database. J Quant Spectrosc Radiat Transfer 2004;88:357-406.

[4] Mishchenko MI, Videen G, Babenko VA, Khlebtsov NG, Wriedt T. Comprehensive T-matrix reference database: a 2004-06 update. J Quant Spectrosc Radiat Transfer 2007;106:304-24.

[5] Mishchenko MI, Videen G, Khlebtsov NG, Wriedt T, Zakharova NT. Comprehensive T-matrix reference database: a 2006-07 update. J Quant Spectrosc Radiat Transfer 2008;109:1447-60.

[6] Mishchenko MI, Zakharova NT, Videen G, Khlebtsov NG, Wriedt T. Comprehensive T-matrix reference database: a 2007-2009 update. J Quant Spectrosc Radiat Transfer 2010;111:650-8.

[7] Barber P, Yeh C. Scattering of electromagnetic waves by arbitrarily shaped dielectric bodies. Appl Opt 1975;14:2864-72.

[8] Varadan VV, Lakhtakia A, Varadan VK. Comments on recent criticism of the T-matrix method. J Acoust Soc Am 1988;84:2280-4.

[9] Bruning JH, Lo YT. Multiple scattering of EM waves by spheres Multipole expansion and ray-optical solutions. IEEE Trans Antennas Propag 1971;19:378-90.

[10] Bruning JH, Lo YT. Multiple scattering of EM waves by spheres II Numerical and experimental results. IEEE Trans Antennas Propag $1971 ; 19: 391-400$.

[11] Peterson B, Ström S. T matrix for electromagnetic scattering from an arbitrary number of scatterers and representations of $\mathrm{E}(3)^{*}$. Phys Rev D 1973;8:3661-78.

[12] Varadan VK, Varadan VV, editors. Acoustic, electromagnetic and elastic wave scattering-focus on the $T$-matrix approach. Pergamon Press: New York; 1980.

[13] Tsang L, Kong JA, Shin RT. Theory of microwave remote sensing New York: Wiley; 1985.

[14] Wiscombe WJ, Mugnai A. Single scattering from nonspherical Chebyshev particles: a compendium of calculations. Washington, DC: National Aeronautics and Space Administration; 1986.

[15] Barber PW, Hill SC. Light scattering by particles: computational methods. Singapore: World Scientific; 1990.

[16] Chew WC. Waves and fields in inhomogeneous media. New York: Van Nostrand Reinhold; 1990.

[17] Varadan VV, Lakhtakia A, Varadan VK. Field representations and introduction to scattering. Amsterdam: North-Holland; 1991. 
[18] Hovenier JW, editor. Light scattering by non-spherical particles. J Quant Spectrosc Radiat Transfer 1996;55:535-694.

[19] Mishchenko MI, Travis LD, Mackowski DW. T-matrix computations of light scattering by nonspherical particles: a review. J Quant Spectrosc Radiat Transfer 1996;55:535-75.

[20] Muinonen K, Nousiainen T, Fast P, Lumme K, Peltoniemi JI. Light scattering by Gaussian random particles: ray optics approximation. J Quant Spectrosc Radiat Transfer 1996;55:577-601.

[21] Hovenier JW, van der Mee CVM. Testing scattering matrices: a compendium of recipes. J Quant Spectrosc Radiat Transfer 1996;55:649-61.

[22] Mishchenko MI. Light scattering by randomly oriented axially symmetric particles. J Opt Soc Am A 1991;8:871-82 (errata: 1992; 9:497).

[23] Mackowski DW, Mishchenko MI. Calculation of the $T$ matrix and the scattering matrix for ensembles of spheres. J Opt Soc Am A 1996;13:2266-78.

[24] Doicu A, Eremin A, Wriedt T. Acoustic and electromagnetic scattering analysis using discrete sources. San Diego: Academic Press; 2000.

[25] Mishchenko MI, Travis LD, Lacis AA. Scattering, absorption, and emission of light by small particles. Cambridge, UK: Cambridge University Press; 2002 (http://www.giss.nasa.gov/staff/mmishchen ko/books.html)

[26] Gumerov NA, Duraiswami R. Fast multipole methods for the Helmhotlz equation in three dimensions. Amsterdam: Elsevier; 2004.

[27] Martin PA. Multiple scattering: interaction of time-harmonic waves with N obstacles. Cambridge: Cambridge University Press; 2006.

[28] Doicu A, Wriedt T, Eremin YuA. Light scattering by systems of particles. Null-field method with discrete sources: theory and programs. Springer: Berlin; 2006.
[29] Borghese F, Denti P, Saija R. Scattering from model nonspherical particles. Theory and applications to environmental physics. Berlin: Springer; 2007.

[30] Rother T. Electromagnetic wave scattering on nonspherical particles: basic methodology and simulations. Berlin: Springer; 2009.

[31] Mishchenko MI, Travis LD, Macke A. T-matrix method and its applications. In: Mishchenko MI, Hovenier JW, Travis LD, editors. Light scattering by nonspherical particles: theory, measurements, and applications. San Diego: Academic Press; 2000. p. 147-72.

[32] Farafonov VG, Il'in VB. Single light scattering: computational methods. In: Kokhanovsky AA, editor. Light scattering reviews. Chichester, UK: Praxis; 2006. p. 125-77.

[33] Wriedt T. Review of the null-field method with discrete sources. J Quant Spectrosc Radiat Transfer 2007;106:535-45.

[34] Wriedt T. Studies of light scattering by complex particles using the null-field method with discrete sources. Light Scattering Rev 2007;2:269-94.

[35] Kahnert M. Light scattering by particles with boundary symmetries. Light Scattering Rev 2008;3:69-107.

[36] Doicu A, Schuh R, Wriedt T. Scattering by particles on or near a plane surface. Light Scattering Rev 2008;3:109-30.

[37] Mishchenko MI. Multiple scattering, radiative transfer, and weak localization in discrete random media: unified microphysical approach. Rev Geophys 2008;46:RG2003.

[38] Okada Y, Kokhanovsky AA. Light scattering and absorption by densely packed groups of spherical particles. J Quant Spectrosc Radiat Transfer 2009;110:902-17.

[39] Rother T. Green functions for plane wave scattering on single nonspherical particles. Light Scattering Rev 2009;4:121-66.

[40] Khlebtsov NG, Dykman LA. Optical properties and biomedical applications of plasmonic nanoparticles. J Quant Spectrosc Radiat Transfer 2010;111:1-35. 